\title{
Luces y sombras del turismo de cruceros: el caso de Barcelona
}

\author{
Luis Alfonso Garay Tamajón
}

Universitat Oberta de Catalunya (UOC). Departamento de Estudios de Economía y Empresa lgaray@uoc.edu

Recepción: diciembre de 2014

Aceptación: mayo de 2015

\section{Resumen}

El objetivo de esta investigación es estimar los impactos económicos y sociales del turismo de cruceros. Para ello, se presenta la evolución de este turismo en la ciudad de Barcelona en las últimas décadas y se examina la colaboración entre las instituciones públicas y privadas en la promoción y en las inversiones que se han realizado en los últimos años para mejorar la ciudad urbana y la infraestructura del puerto de cruceros. Sin embargo, el espectacular crecimiento de este tipo de turismo, que ha colocado a la ciudad de Barcelona como un líder mundial en este sector, ha provocado una serie de impactos en el destino, positivos y negativos. Por ello, este trabajo también analiza cuál es la percepción que tienen los residentes respecto a este turismo, lo cual es muy importante para planificar la gestión estratégica de un desarrollo sostenible del turismo de cruceros en Barcelona.

Palabras clave: turismo de cruceros; turismo urbano; puerto; impactos; Barcelona; turismo sostenible.

Resum. Llums i ombres del turisme de creuers: el cas de Barcelona

L'objectiu d'aquesta recerca és estimar els impactes econòmics i socials del turisme de creuers. Per a això, es presenta l'evolució d'aquest turisme a la ciutat de Barcelona en les últimes dècades i s'examina la col-laboració entre les institucions públiques i privades en la promoció i en les inversions que s'han realitzat en els últims anys per millorar la ciutat urbana i la infraestructura del port de creuers. No obstant això, l'espectacular creixement d'aquest tipus de turisme, que ha col-locat la ciutat de Barcelona com un líder mundial en aquest sector, ha provocat una sèrie d'impactes en la destinació, positius i negatius. Per això, aquest treball també analitza quina és la percepció que tenen els residents respecte a aquest turisme, la qual cosa és molt important per planificar la gestió estratègica d'un desenvolupament sostenible del turisme de creuers a Barcelona.

Paraules clau: turisme de creuers; turisme urbà; port; impactes; Barcelona; turisme sostenible. 
Résumé. Nouveaux tourismes dans la ville de Barcelone: le tourisme de croisières

L'objectif de cette recherche est d'estimer les impacts économiques et sociaux du tourisme de navires de croisière. Pour cela, nous présentons l'évolution de ce tourisme dans la ville de Barcelone lors des dernières décennies et nous examinons la collaboration entre les institutions publiques et privées dans la promotion et dans les investissements réalisés lors des dernières années pour améliorer la ville urbaine et l'infrastructure du port de navires de croisière. Cependant, la croissance spectaculaire de ce type de tourisme qui a placé la ville de Barcelone en position d'un leader mondial dans ce secteur a provoqué une série d'impacts, positifs et négatifs, sur cette destination. Pour cette raison, ce travail analyse aussi la perception que les résidents ont de ce tourisme, aspect très important pour planifier la gestion stratégique d'un développement durable du tourisme de navires de croisière à Barcelone.

Mots-clés: tourisme de navires de croisière; tourisme urbain; port; impacts; Barcelone; tourisme durable.

\section{Abstract. New tourism in the city of Barcelona: Cruise tourism}

The aim of this investigation is to estimate the economic and social impacts of cruise tourism on Barcelona. For this purpose, we examine the development and evolution of cruise tourism in the city in recent decades, as well as public and private collaboration in recent years to promote and invest in improving the urban city and cruise port infrastructure. Although the spectacular growth of cruise tourism has positioned Barcelona as a world leader in this sector, it has impacted on the destination both positively and negatively. Residents' perceptions regarding this type of tourism are also discussed as they are key to the strategic management planning and sustainable development of cruise tourism in Barcelona.

Keywords: cruise tourism; urban tourism; port; impacts; Barcelona; sustainable tourism.

\section{Sumario}

1. Introducción $\quad 5$. Percepción de la población de

2. El turismo de cruceros. Estado de la cuestión y evolución

3. Metodología

4. El desarrollo del turismo de cruceros en

Barcelona y la cooperación público-privada
Barcelona respecto a los impactos del turismo de cruceros

6. Conclusiones

Referencias bibliográficas

\section{Introducción}

El turismo de cruceros permite viajar entre distintos puertos y países, con itinerarios predefinidos y una oferta en régimen de "todo-incluido», de modo que en el mismo espacio físico se fusionan transporte, alojamiento y ocio (Tang y Jang, 2013). Sin embargo, aunque esta actividad puede entenderse como un resort en movimiento, es evidente su influencia en los ámbitos económico, 
sociocultural y medioambiental de los destinos y, muy especialmente, en los puertos base (destinos origen y final de trayecto).

Barcelona se ha convertido en el principal puerto europeo de cruceros del Mediterráneo y en el cuarto a escala internacional, sobrepasado únicamente por los de Miami, Port Canaveral y Port Everglades, todos ellos en Florida (Estados Unidos de América). Entre las seis terminales del puerto barcelonés se han recibido 2,6 millones de cruceristas en el año 2013 y 2,7 millones en el 2014 (Hosteltur, 2014). Las perspectivas son de mantener e incluso aumentar este número en los próximos años.

El crecimiento que ha experimentado este turismo en Barcelona en las últimas décadas ha provocado una serie de impactos positivos y negativos en la ciudad. Por ejemplo, ha ayudado a que se modificasen algunas de las pautas tradicionales, especialmente en el sector comercial, con decisiones más o menos controvertidas como la apertura de los establecimientos comerciales en días festivos. Por ello, una de las críticas a este producto es que su desarrollo debería asentarse en unas bases sostenibles, con una oferta de calidad que no solo esté preocupada por el beneficio económico inmediato sino que también presente criterios de responsabilidad social y ambiental cuantificables y demostrables.

Ante esta situación, el objetivo de este trabajo es triple. Por una parte, describir la evolución del turismo de cruceros en Barcelona y conocer qué organizaciones han sido las protagonistas de este desarrollo desde el ámbito público y privado. Por la otra, conocer cuál es la percepción que los residentes en esta ciudad tienen sobre los posibles impactos positivos y negativos que genera este producto. Finalmente, un tercer objetivo es ver qué riesgos puede comportar este turismo. Por este motivo, después de presentar la evolución del turismo de cruceros, especialmente en Barcelona, se han comprobado las tres hipótesis siguientes:

a) La conjunción de una correcta colaboración entre las instituciones públicas y privadas, tanto en la promoción del destino y del producto como en la realización de las necesarias infraestructuras urbanas y portuarias han sido fundamentales para el desarrollo de este turismo (hipótesis 1).

b) Los residentes perciben que las ventajas que reporta esta actividad son mayores que sus desventajas, por lo que su actitud suele ser favorable a este tipo de turismo (hipótesis 2).

c) A pesar de esta percepción positiva, ya empiezan a aparecer algunos interrogantes para el futuro, empezando por su sostenibilidad (hipótesis 3).

\section{El turismo de cruceros. Estado de la cuestión y evolución}

$\mathrm{Al}$ tratarse de un tipo de turismo emergente, la literatura científica existente al respecto es todavía bastante escasa y se retrotrae solo hasta finales de los años ochenta. Así, entre los primeros trabajos académicos publicados destacan los de Lawton y Butler (1987), Dwyer y Forsyth (1998), Henthorne (2000), Wood (2000) y Timothy, (2006). Sin embargo, todos ellos están centrados 
en el mercado norteamericano y el destino del Caribe, ya que hasta entonces ambos habían sido absolutamente predominantes.

En Europa, poco a poco, también se ha ido creando una bibliografía dedicada a analizar este fenómeno turístico, sobre todo en estos últimos veinte años, con artículos y obras de autores como Montero (1996), Esteve (1998), Kester (2002), Murias (2002), Duman y Mattila (2005), Brida y Zapata (2008), Cuellar-Río y Kido-Cruz (2008), Silvestre, Santos y Ramalho (2008), Andriotis y Agiomirgianakis (2010), Brida, Riaño y Zapata (2012), Garay y Cànoves (2012), Brida, Pulina, Riaño y Zapata (2013), Legoupil (2013) y Torres y Nieto (2014).

Aunque el fenómeno del crucerismo tal y como se le conoce hoy en día es relativamente contemporáneo (años sesenta del siglo xx), los primeros ejemplos de este turismo se iniciaron en la década de los años treinta del siglo XIx, con ofertas en Hamburgo (Alemania) de viajes de ocio para familias adineradas (Murías, 2002). Posteriormente, a mediados del mismo siglo se fundó la Cunard Line, una de las grandes líneas de transatlánticos que unieron Europa y América (Torres y Nieto, 2014), y Thomas Cook creó las primeras agencias de viajes con sus cruceros elitistas a Egipto (Towner, 1985). En 1858 se fundó la compañía británica Peninsular and Oriental Steam Ship Navigation Company (P\&O). El éxito de estas iniciativas se basaba en la reproducción de las primeras actividades vinculadas al turismo en sí, ya que seguían el itinerario que habían realizado décadas antes los viajeros del "Grand Tour», con destinos como Italia, Grecia o Egipto (Buzard, 1993).

Esta primera época dorada de los cruceros finalizó en las primeras décadas del siglo XX con las dos guerras mundiales, cuando la mayor parte de los buques fueron destinados al transporte de tropas o servicios logísticos. Las consiguientes posguerras no ofrecieron tampoco un contexto favorable para esta actividad, ya que la mayoría de los buques estaban hundidos o fuertemente dañados.

Posteriormente, entre los años cincuenta y sesenta del siglo pasado, se presentó una conjunción de elementos que hizo posible su renacimiento. Por un lado, emergió y se consolidó una demanda que en principio nadie preveía, con lo que se popularizó y masificó el fenómeno turístico para convertirse en uno de los mejores ejemplos de la etapa "fordista». Esta creciente demanda hizo que los turistas pasaran de desplazarse en tren a hacerlo en automóvil («motorización») y posibilitó el auge de la aviación comercial. En este contexto, las compañías navieras que realizaban viajes transatlánticos decidieron transformar sus buques de línea convencionales en centros de ocio con todo tipo de servicios para un turista inicialmente con un perfil de poder adquisitivo alto y medio-alto (Tang y Jang, 2013) y, después, con el modelo de "todo incluido», que ha triunfado en el sector.

Así se inició la definitiva popularización del turismo de cruceros, con compañías tan reconocidas como Carnival, Royal Caribbean, Cruise Line o Princesa (Fernández Fúster, 2009). Además, el progresivo aumento de la capacidad de los buques permitió transportar más pasajeros y obtener más ingresos (Timothy, 2006), aunque este mayor tamaño necesitaba altas inversiones, al alcance de pocas compañías, por lo que finalmente se consolidaron internacionalmente 
solamente tres grandes grupos navieros (Carnival Corporation, Royal Caribbean y Star Cruises), que controlaban el 88\% de la oferta mundial (OMT, 2008). Paralelamente, aparecieron grandes asociaciones, como la americana Cruise Lines International Association o la europea Cruise Europe, cuyo papel fue clave para canalizar la relación entre compañías y puertos (Murias, 2002).

Este desarrollo de la oferta estuvo acompañado por un gran crecimiento de la demanda, especialmente en las dos últimas décadas. Tras su desarrollo en los Estados Unidos, Europa se añadió como mercado emisor y, entre 1999 y 2011, pasó de 1,9 a 5,6 millones de cruceristas, con lo que alcanzó el 30\% de la demanda mundial (Consejo Europeo de Cruceros, 2012).

Posteriormente, en 2012, los puertos europeos recibieron 29,3 millones de viajeros (lo que representó una tasa media de crecimiento del 10\% en diez años), de modo que en cuarenta años la demanda mundial se ha multiplicado cuarenta veces (Euroxpress, 2014). Aun sí, el mercado norteamericano sigue dominando, con 10,92 millones de viajeros, seguido del Reino Unido e Irlanda $(1,73$ millones); Alemania $(1,69)$; Italia $(0,87)$; Australia $(0,83)$; Canadá $(0,77)$; China $(0,73)$ y Brasil $(0,73)$, según el último informe de la Cruise Lines International Association (CLIA, 2014).

El principal destino mundial sigue siendo el Caribe, especialmente durante la época invernal, ya que recibe gran parte de la demanda norteamericana (CLIA, 2014). Ahora bien, el Mediterráneo es el destino que más está creciendo en estos últimos años, con una demanda que se reparte entre los mercados europeo y norteamericano. En todo caso, aún existe un gran potencial para este sector, especialmente en algunos países asiáticos, y se estima un mercado de cerca de treinta millones de turistas en los próximos años (CLIA, 2014).

En cuanto a ingresos, este crecimiento ha sido parejo. Así, en 2013, el negocio directo generado por este producto turístico alcanzó los 15.500 millones de euros, lo que representó el doble que diez años atrás (Euroxpress, 2014), y generó 891.000 puestos de trabajo a tiempo completo (CLIA, 2014).

En España, aunque con un cierto retraso en el tiempo, también se ha producido el mismo fenómeno evolutivo. Así, en 1930, la compañía naviera Ybarra puso en funcionamiento el buque Cabo San Antonio con una doble finalidad: cabotaje y transporte de pasajeros (Castillo e Ybarra, 2004). El número de viajeros turísticos siguió siendo escaso a pesar del impulso de organizaciones como la Sociedad de Atracción de Forasteros (Garay y Cànoves, 2012). Además, la situación social del momento en el país no favorecía tampoco la llegada de este tipo de turistas. Esta incipiente actividad se interrumpió bruscamente como consecuencia de la Guerra Civil (1936-1939) y de la posguerra, hasta que con el fin del periodo autárquico y la apertura internacional empezó el fenómeno turístico, que constituiría uno de los puntales del desarrollismo español.

En 1961 la naviera Ybarra empezó a realizar viajes solo de pasajeros hacia Sudamérica, y otras empresas programaron cruceros turísticos de más corta duración (Castillo e Ybarra, 2004). La emergencia de un nuevo crucerismo se sitúa en paralelo a la explosión del turismo de sol y playa en el país, aunque con peculiaridades todavía del ocio "prefordista». Eran viajes pensados para 
un público de alto poder adquisitivo y que disponía de suficiente tiempo para un viaje largo de estas características, por lo que solamente 20.000 españoles hacían vacaciones anualmente en un crucero, cifra que se mantuvo casi constante hasta mediados de los años noventa, en los que comenzó un crecimiento sostenido de pasajeros turísticos que llevaría a las cifras actuales (Paniagua, 2005).

No se puede olvidar que en la década de 1970, las sucesivas crisis del petróleo provocaron una importante alza del precio de este combustible, lo que produjo una fuerte crisis en el sector que hizo reducir significativamente la flota de buques. Además, la competencia de la compañía Transmediterránea, empresa controlada y subvencionada por el Estado, era cada vez mayor, ya que podía trabajar con déficit (Castillo e Ybarra, 2004).

En los últimos años se ha producido la explosión del fenómeno crucerista, de modo que la emisión de turistas de cruceros españoles se ha multiplicado por cinco y la recepción ha superado los cinco millones, con lo que se ha consolidado como el cuarto mercado europeo. El puerto de Barcelona, que quintuplicó su demanda entre 1995 y 2005, es el líder de la región mediterránea, y entre los puertos de Barcelona y Palma de Mallorca se concentra el 80\% de las salidas, mientras que el $75 \%$ de las escalas se lleva a cabo en las Canarias, las Baleares, Barcelona y Málaga.

Así pues, el turismo de cruceros resulta cada vez más interesante para muchas ciudades portuarias, ya que además de los ingresos directos también genera otros adicionales durante las visitas de estos turistas, especialmente en los puertos base (pernoctaciones hoteleras, excursiones, compras, consumos en bares y restaurantes, entradas para espectáculos, transportes, etc.). Además, como que el crucerista hace escalas rápidas en las ciudades de la ruta, si percibe que estas tienen un cierto interés para él, puede decidir volver a visitarlas por su cuenta en el futuro, de un modo más pausado y profundo. Hay que tener en cuenta que el perfil medio de este tipo de turista es una persona de mediana edad (alrededor de 45 años), con formación media o superior e ingresos medio-altos y altos, aunque en los últimos años han empezado a crecer otros segmentos, como el joven y la gente mayor (APB, 2006-2009).

Ante esta situación, la oferta ha desarrollado un producto más a la medida de cada segmento. Así, el «Budget», que se corresponde con la clase más económica, utiliza barcos antiguos reformados y con menores servicios. El "Contemporary», que es el predominante (con más de la mitad del mercado), usa buques de mediano o gran tamaño, con unos servicios e infraestructuras notables. Por su parte, el «Premium», de mayor calidad, ofrece un muy buen nivel de servicios. Finalmente, el «Luxury» son auténticos hoteles de lujo en el mar, en los que embarcar puede suponer más mil dólares diarios para el turista.

Por otro lado, tomando como consideración previa que la demanda de tipo genérico aún sigue siendo mayoritaria, se están empezando desarrollar los cruceros temáticos o especializados, los conocidos como "Niche Cruises», que tienen como eje vertebrador alguna actividad concreta (por ejemplo, las escalas en destinos relacionados con el golf, destinos románticos, activos, etc.) o la visita a lugares difícilmente accesibles para el turismo más convencional. 
En el mundo actual, globalizado gracias a la informática, los transportes y las comunicaciones, los turistas, cada vez con mayor poder adquisitivo y tiempo libre, son más experimentados y exigentes, y demandan no solo un producto de una cierta calidad y a un precio asequible, sino también un trato diferenciado y unas actividades personalizadas (Prat y Cànoves, 2012). El turismo de cruceros sigue estas mismas tendencias: ofrece experiencias de viaje interesantes y variadas, con trayectos cortos, especializados y adaptados a las disponibilidades de tiempo libre de la sociedad actual, abarata el paquete contratado y puede acceder a mayores segmentos de demanda (Lagoupil, 2013).

Según la OMT (2008), el futuro del turismo de cruceros exige una mayor innovación y flexibilidad en la oferta, el atraque de buques cada vez mayores, la mejora de los servicios y la mayor seguridad posible. En cuanto a la demanda, los cambios sociodemográficos (envejecimiento de la población, diferentes unidades familiares, segmentación de las vacaciones), el proceso de globalización, el cambio climático o el uso habitual de las tecnologías de la información y la comunicación también desempeñarán un papel importante (OMT, 2008).

Sin embargo, también resulta evidente que los aspectos relacionados con la sostenibilidad serán claves en el futuro de este negocio, ya que existen impactos peligrosos para este sector. Uno de ellos es la necesidad de un correcto control medioambiental. Así, por ejemplo, los niveles de contaminación del aire provocados actualmente por la llegada de grandes naves de crucero a las terminales de los puertos resultan preocupantes, como lo demuestran las mediciones realizadas periódicamente en Barcelona por diversas asociaciones de vecinos y grupos ecologistas (Nueva Tribuna, 2014). No hay que olvidar que los cruceros utilizan fueloil pesado, que contiene hasta 3.500 veces más azufre que el diésel de los automóviles y camiones, y la quema de este aceite residual emite altas cantidades de contaminantes tóxicos en el aire, como el dióxido de nitrógeno, partículas, dióxido de sulfuro y otros hidrocarburos peligrosos. Sus emisiones afectan a la salud humana, la agricultura, los ecosistemas y contribuyen al cambio climático. Además, las naves mantienen sus motores trabajando a baja intensidad para mantener todos sus sistemas eléctricos funcionando mientras están atracadas en el puerto.

Otros aspectos que presentan importantes factores de riesgo son: la baja calidad de los puestos de trabajo que genera este tipo de actividad; la falta de afiliación sindical de las tripulaciones (salarios bajos y limitados derechos laborales); la futura regulación medioambiental marina europea (que puede limitar el tamaño de los buques o la frecuencia de los trayectos); la necesidad constante de innovación en materia logística; y la falta de una adecuada interconexión nodal con los restantes medios de transporte de pasajeros (ITF, 2014).

\section{Metodología}

Para comprobar la primera de las tres hipótesis aquí propuestas, teniendo en cuenta la literatura anteriormente citada, se ha seguido una metodología cualitativa, basada fundamentalmente en la revisión bibliográfica, y otra más cuantitativa, basada en datos estadísticos. 
Tabla 1. Cuestiones planteadas

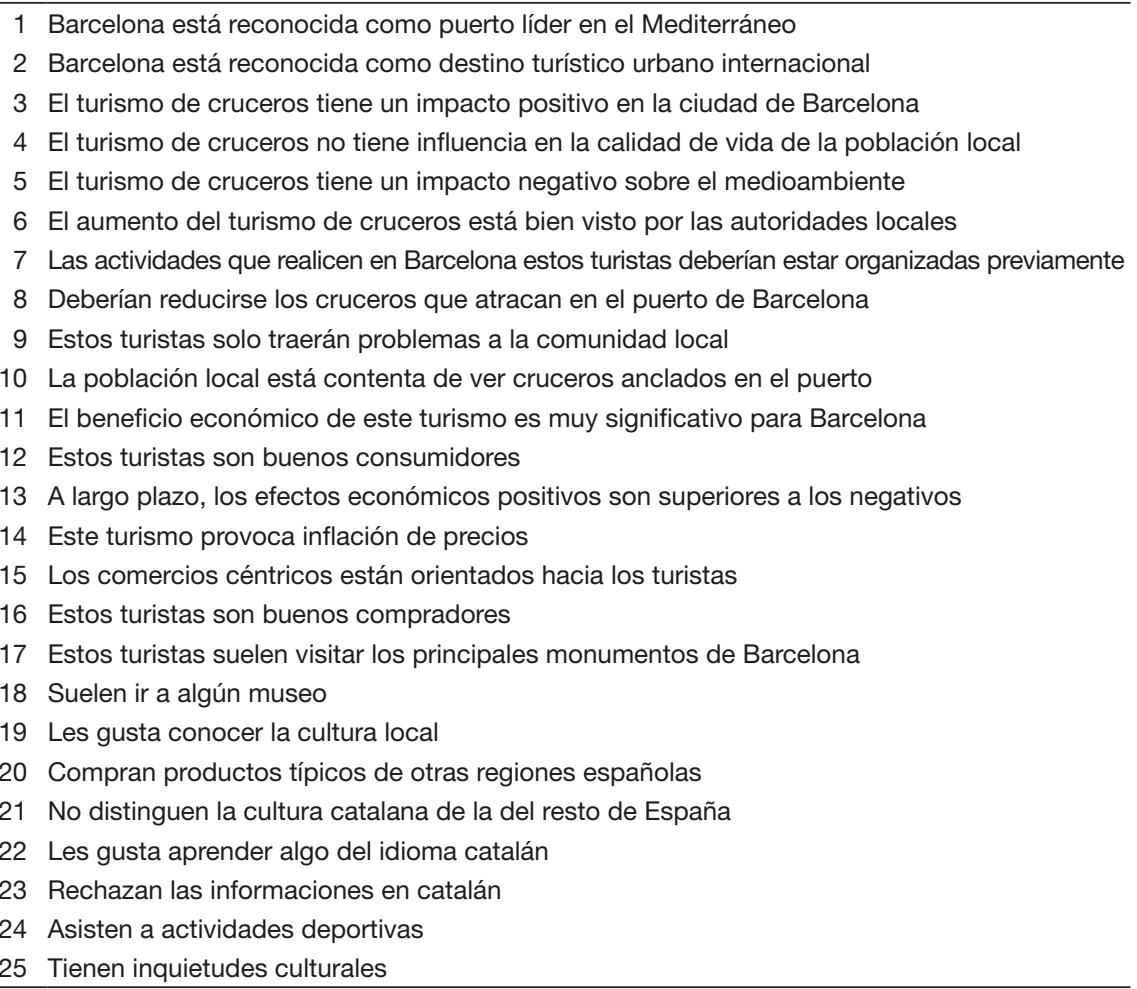

Fuente: elaboración propia.

Para confirmar la segunda, se ha realizado una encuesta personal al azar a residentes en Barcelona, algunos con algún tipo de relación con el turismo y otros no. Para ello, a partir de las indicaciones de Harril (2004) y de las variables propuestas por Monterrubio (2008) y Castillo et al. (2012), se ha diseñado un cuestionario con veinticinco preguntas (Tabla 1). Su valoración era del 1 al 5, según la escala de Likert (1 = muy en desacuerdo; $5=$ muy de acuerdo). De estas veinticinco preguntas, diez estaban relacionadas con aspectos socioambientales (de la 1 a la 10), otras seis con aspectos económicos (de la 11 a la 16) y las restantes nueve con aspectos culturales (de la 17 a la 25).

Dicha encuesta se realizó en el puerto de Barcelona y sus aledaños durante el mes de diciembre de 2013 por personal de nuestro grupo de investigación, que inicialmente contactó con 120 personas, de las que 60 estaban relacionadas de algún modo con el sector turístico y las otras 60, no. Aceptaron responder a las preguntas 84 personas (el 70\% de los contactos realizados), de las que 48 tenían algún tipo de relación con el turismo y 36 no tenían ninguna. 
Tabla 2. Fiabilidad test-retest de las preguntas seleccionadas

\begin{tabular}{cccccc} 
N. ${ }^{\circ}$ pregunta & $\begin{array}{c}\text { Coeficiente } \\
\text { kappa }\end{array}$ & N. ${ }^{\circ}$ pregunta & $\begin{array}{c}\text { Coeficiente } \\
\text { kappa }\end{array}$ & N. ${ }^{\circ}$ pregunta & $\begin{array}{c}\text { Coeficiente } \\
\text { kappa }\end{array}$ \\
\hline 1 & 0,63 & 10 & 0,65 & 19 & 0,80 \\
2 & 0,73 & 11 & 0,58 & 20 & 0,92 \\
3 & 0,81 & 12 & 0,59 & 21 & 0,83 \\
4 & 0,76 & 13 & 0,65 & 22 & 0,74 \\
5 & 0,65 & 14 & 0,69 & 23 & 0,70 \\
6 & 0,59 & 15 & 0,78 & 24 & 0,62 \\
7 & 0,77 & 16 & 0,86 & 25 & 0,74 \\
8 & 0,87 & 17 & 0,82 & & \\
9 & 0,82 & 18 & 0,91 & & \\
\hline
\end{tabular}

Fuente: elaboración propia.

Para valorar el grado de fiabilidad de esta encuesta como instrumento de medida, se ha calculado el coeficiente kappa (Armitage y Berry, 1997), que ha sido posiblemente el método de test-retest generalmente más utilizado (Comín, 1990). Dicho coeficiente oscila entre 0 y 1 , de tal forma que el valor 1 corresponde a una concordancia total y el valor 0 a una concordancia nula; se considera un grado de fiabilidad malo cuando dicho coeficiente oscila entre 0 y 0,4 ; aceptable si está entre 0,4 y 0,75 , y bueno si es superior a 0,75 . En el caso presente, los resultados obtenidos para el coeficiente kappa (Tabla 2) muestran que trece preguntas tenían una fiabilidad aceptable y las otras doce una fiabilidad buena. Ninguna presentaba un grado de fiabilidad mala, lo que permite confirmar la fiabilidad de la encuesta.

Una vez realizada la encuesta se comprobó que el perfil sociodemográfico de los 84 encuestados era, en cuanto al género, de 45 hombres (el 53,57\%) y 39 mujeres (el 46,43\%). De ellos, 56 residían en la propia ciudad de Barcelona (el 66,67\%) y los restantes 28 (el 33,33\%) lo hacían en su región metropolitana. Por grupos de edad, 36 tenían entre 18 y 39 años (el 42,86\%), 35 entre 40 y 59 años (el 41,67\%) y 13 superaban los 60 años (el 15,47\%). Respecto a su nivel de estudios, 44 habían realizado solamente la formación básica (el 52,38\%), 10 tenían formación universitaria (el 11,91\%) y el resto estaba realizando los estudios secundarios (el 35,71\%). En el ámbito laboral, 12 trabajaban en el sector público (el 14,29\%), 48 en el privado (el 57,14\%) y los 24 restantes eran jubilados, amas de casa, estudiantes o desempleados. De los 48 que trabajaban en actividades relacionadas con el turismo, solamente 22 lo hacían directamente en el turismo de cruceros. En cuanto al nivel de ingresos, 79 encuestados ganaban menos de 2.000 euros al mes (el 94,05\%).

En lo referente a la tercera hipótesis planteada, para su comprobación se ha utilizado parcialmente la anterior encuesta, así como datos cuantitativos y la bibliografía existente sobre este tema. 


\section{El desarrollo del turismo de cruceros en Barcelona y la cooperación público-privada}

En el siglo XIX ya existían navieras que operaban en el puerto de Barcelona y que, además del transporte de mercancías, también realizaban transportes regulares de viajeros, casi siempre relacionados con el desplazamiento de emigrantes desde la Península hacia las colonias de ultramar y los países latinoamericanos, como en el caso de Ybarra (Castillo e Ybarra, 2004). A finales del mismo siglo se produjo una gran transformación del puerto barcelonés, con la construcción del primer muelle transversal, dirigida por la Junta de Obras del Puerto de Barcelona. Empezaron a operar compañías como la Transatlántica y la Transmediterránea, ambas dedicadas ya al transporte de viajeros, aunque el porcentaje de turistas era todavía muy escaso.

En los dos primeros tercios del siglo pasado, el puerto de Barcelona, con un carácter eminentemente industrial, continuó creciendo al amparo de nuevos proyectos, como el Plan General de Desarrollo (1965-1966), y se extendió hacia el delta del Llobregat con la construcción de un puerto interior junto al río (Alemany, 2002).

Con la llegada de la democracia a España se produjeron importantes cambios que afectarían al turismo de cruceros en el puerto barcelonés. Así, en 1978 se conseguía el Estatuto de Autonomía del Puerto, lo que permitió su desarrollo en tres zonas al ampliar de nuevo sus infraestructuras hacia el delta del Llobregat: el Port Vell, el puerto comercial y el puerto logístico.

En los años ochenta, a pesar de que empezaron a aparecer en España turoperadores como Unión Lloyd, la Central de Cruceros o Latitud 4, el puerto de Barcelona no era todavía un destino de cruceros demasiado reconocido internacionalmente, aunque algunas navieras españolas, como Transmediterránea e Ybarra, lo mantuvieron como puerto base con viajes de transporte regulares, que no respondían al perfil de crucero, especialmente a Génova y Palma de Mallorca.

La situación cambió con motivo de la celebración de los XXV Juegos Olímpicos en Barcelona, en julio de 1992. Dicho evento sirvió para que la ciudad acometiese una profunda transformación de sus infraestructuras y de su propia imagen (Garay y Cànoves, 2009). La colaboración entre el Ayuntamiento - representado por el Patronato Municipal de Turismo-y el Comité de Turismo de la Cámara de Comercio, Industria y Navegación de Barcelona dio como fruto el plan estratégico, cuyo objetivo primordial era situar la ciudad como un importante destino turístico internacional, aprovechando el impacto de los Juegos. Pasados estos, en 1993, se constituyó un organismo de promoción turística (Turisme de Barcelona), centrado en la promoción específica y la especialización turística a partir de la identificación de diferentes segmentos de mercado, entre los que se encontraba el turismo de cruceros.

Para los Juegos Olímpicos se realizaron importantes actuaciones urbanísticas, como la apertura de la ciudad al mar, la remodelación del Port Vell, la construcción de nuevos barrios marítimos y la disponibilidad de espacios 
públicos — playas y paseos-, necesarias para acoger la creciente demanda de turistas que después ha tenido lugar. Equidistante geográficamente de las grandes aglomeraciones turísticas de sol y playa de la Costa Brava y de la Costa Dorada, el patrimonio cultural de la capital catalana se ponía en valor para un público internacional que redescubría sus atractivos tras muchas décadas de letargo. Así, entre 1991 y 2005 el número de turistas que pernoctaban en la capital catalana prácticamente se triplicó, y pasó de poco más de un millón setecientos mil a más de cinco millones (AB, 1992-2011).

Paralelamente, el órgano gestor Port de Barcelona, rebautizado como Autoritat Portuària de Barcelona (APB), empezó a desarrollar, en el marco del plan estratégico, las infraestructuras portuarias adecuadas para conseguir este objetivo, ya que entendió las potencialidades que ofrecía este tipo de turismo para la ciudad e impulsó un tráfico de pasajeros que, en principio, no debía suponer un incremento importante de inversiones en infraestructuras. De esta manera se diseñó un plan especial, consensuado con la Administración municipal, con el objetivo de reordenar el Port Vell e incorporar nuevas terminales para líneas regulares y de cruceros, de modo que al finalizar la década de los noventa el puerto ya tenía cinco estaciones marítimas para cruceros y dos que se podían intercambiar con los ferris.

El crecimiento de este turismo se ha puesto de manifiesto especialmente en estos últimos años (Figura 1), con más de 2,65 millones de pasajeros en el 2011, 2,4 en el 2012, 2,6 en el 2013 y una estimación de 2,7 en el 2014 (Hosteltur, 2014), teniendo en cuenta que en el año 2013 el puerto de Barcelona recibió nuevas escalas de cinco compañías navieras (Carnaval Sunshine, MSC Preziosa, Carnaval Legend, Royal Princesa y Compagnie des Iles du Ponant).

El auge de este turismo se ha confirmado con la llegada al puerto barcelonés en el 2014 del Oasis of the Seas, de la compañía Royal Caribbean, con 220.000 toneladas, 12.000 pasajeros -entre los que embarcan y desembarcan-y 2.200 tripulantes. Asimismo, para el año 2015 está prevista la llegada del crucero más grande del mundo, el Allure of the Seas, también de la compañía Royal

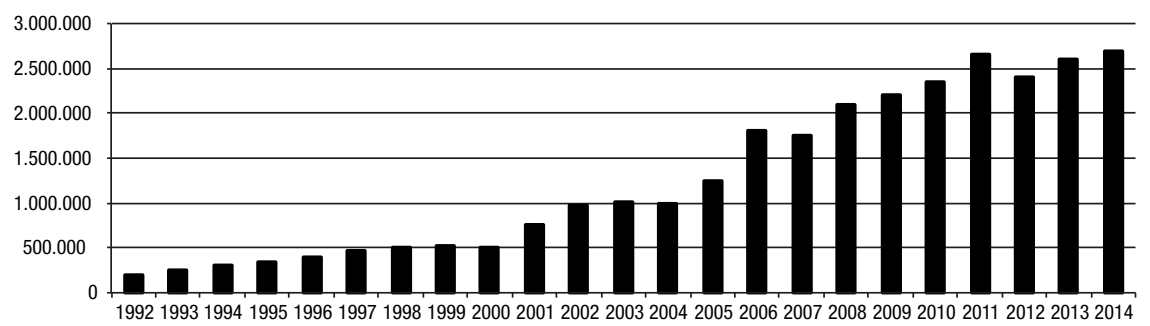

Figura 1. Evolución del número de pasajeros de cruceros en Barcelona (1992-2014).

Fuente: elaboración propia a partir de datos de la Autoritat Portuària de Barcelona cedidos al Ayuntamiento de Barcelona (AB, 1992-2012) y Hosteltur (2014). 
Caribbean, con 25 salidas desde el puerto de Barcelona, un movimiento de 158.000 viajeros y un impacto económico cercano a los 20 millones de euros.

Así pues, el turismo de cruceros se ha consolidado como una importante actividad económica para la ciudad. Un estudio del European Cruise Council (ECC, 2007) sobre el impacto de los cruceros en las economías locales indicaba que ya entonces el negocio de los cruceros en Barcelona era responsable de 14.000 puestos de trabajo, con una remuneración total superior a los 400 millones de euros. Aun así, la Autoritat Portuària de Barcelona considera que, teniendo en cuenta que los cruceros ocupan entre un 10 y un $15 \%$ de la línea de atraque del puerto, tan solo generan un 3,5\% de la cifra de negocio del mismo.

Analizando el gasto que realizan dichos turistas, según Turisme de Barcelona (2014), cada viajero que acaba o inicia ruta en la ciudad condal gasta una media de 123,68 euros al día, mientras que los que solamente hacen escala de unas horas dejan 62 euros, y los tripulantes en sus horas libres (se estima que baja a pasear por la ciudad el 30\%) gastan 23 euros (Tabla 3).

De este modo, según los últimos datos de Turisme de Barcelona (2014), el impacto económico de este turismo ascendió a más de 256 millones de euros durante el año 2013, lo que representa casi 25 millones de euros más que en el año anterior. En esta nueva cifra están incluidos los 2.599.232 pasajeros y los 1.039.693 tripulantes de las embarcaciones que pasaron por Barcelona en dicho año.

Así pues, confirmando la hipótesis 1, se puede concluir que Barcelona se ha convertido en un destino líder del turismo de cruceros a escala internacional, cuya clave de éxito se explica por la eficiente colaboración entre las instituciones públicas y privadas para realizar las inversiones y la promoción necesarias para adaptar las infraestructuras portuarias y urbanas y desarrollar nuevos productos complementarios a partir de una buena segmentación de la demanda. Dichos organismos pueden focalizarse fundamentalmente en la Autoritat Portuària (APB) y el consorcio Turisme de Barcelona, de manera que mientras la primera se ha centrado en atender a los armadores y a las tripulaciones, la segunda se ha ocupado de desarrollar la comunicación turística. Otro elemento clave de la cooperación entre las instituciones públicas y privadas ha sido el desarrollo de una amplia y diversa oferta hotelera, ya que al ser puerto base de

Tabla 3. Impacto económico de los cruceros en Barcelona (2013)

\begin{tabular}{lccc}
\hline \multicolumn{1}{c}{ Pasajeros } & Cantidad & Gasto diario (euros) & Gasto total (euros) \\
\hline En tránsito & 1.092 .946 & 62,00 & 67.762 .652 \\
Puerto base & 1.506 .286 & 123,68 & 182.591 .989 \\
Total pasajeros & 2.599 .232 & & 250.354 .641 \\
\hline Tripulantes & 1.039 .693 & & \\
Tripulantes que visitan la ciudad & 311.908 & 23 & 6.550 .068 \\
\hline Total impacto económico & & & 256.904 .709 \\
\hline
\end{tabular}

Fuente: Turisme de Barcelona, 2014. 
un gran número de trayectos se facilita la pernoctación a muchos turistas que aquí inician o finalizan sus recorridos en barco.

\section{Percepción de la población de Barcelona respecto a los impactos del turismo de cruceros}

En cuanto a la percepción que tienen los residentes sobre los impactos que genera el turismo de cruceros en la ciudad, los resultados de la encuesta realizada muestran una percepción global positiva superior a la negativa, ya que la valoración media de los impactos positivos es de 3,58 (sobre 5), mientras que la de los negativos es de 2,92. Si se desglosan los resultados según el tipo de impacto (socioambiental, económico y cultural) (Figura 2), la percepción media en el caso de los impactos socioambientales positivos es muy superior a la de los negativos $(3,63$ frente a 1,99$)$. Lo mismo ocurre en el caso de los impactos económicos (4,64 frente a 3,46). En cuanto a los impactos culturales, la diferencia se estrecha $(3,97$ frente a 3,50).

Si se analizan las respuestas a cada pregunta del cuestionario en función de si los encuestados estaban relacionados o no con el turismo (Figura 3), entre los residentes está generalizada la percepción de que Barcelona está reconocida como puerto líder en el Mediterráneo (pregunta 1) y como destino turístico urbano de primer orden (pregunta 2). Además, opinan que el turismo de cruceros tiene un impacto positivo para la ciudad (pregunta 3), sin influir en la calidad de vida de la misma (pregunta 4) ni tener un impacto demasiado negativo sobre el medioambiente (pregunta 5), por lo que está bien visto por las autoridades locales (pregunta 6) y la población local (pregunta 10). La percepción de que existe beneficio económico de este turismo para Barcelona se corrobora (preguntas 11 y 13), ya que estos turistas son buenos consumidores (pregunta 12) y compradores (pregunta 16). A pesar de ello, los encuestados creen que existe riesgo de que este turismo ayude a subir los precios de los productos (pregunta 14), ya que muchos comercios céntricos están orientados hacia el turismo (pregunta 15).

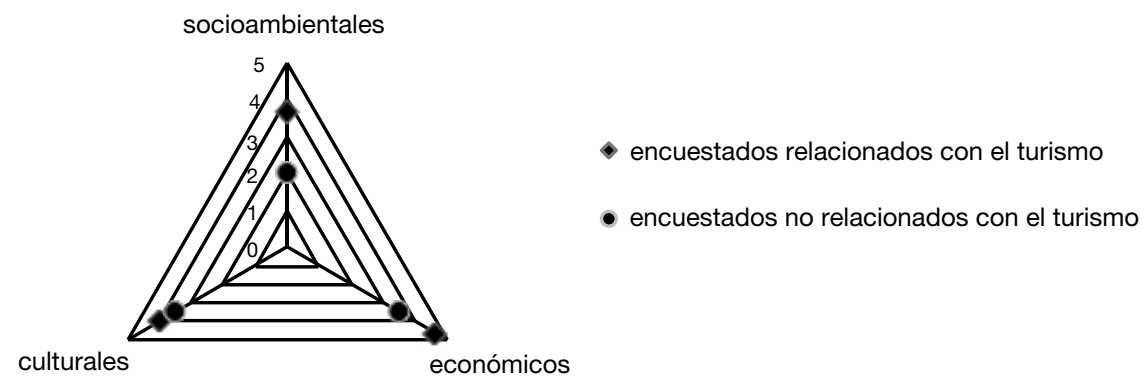

Figura 2. Percepción de los residentes sobre los impactos del turismo de cruceros.

Fuente: elaboración propia. 


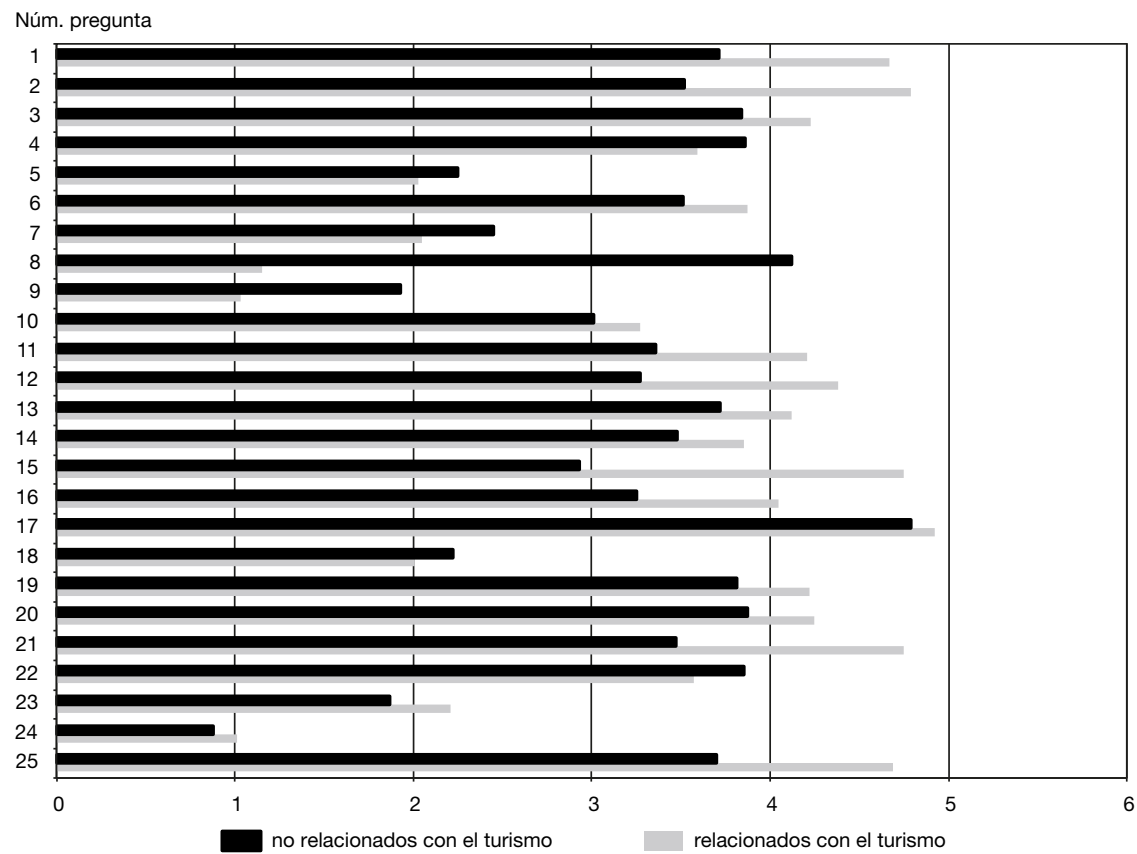

Figura 3. Percepción de los residentes sobre los impactos del turismo de cruceros.

Fuente: elaboración propia.

En el ámbito cultural, los encuestados perciben que a estos turistas les gusta conocer la cultura (pregunta 25) y el idioma local (preguntas 19, 22 y 23) y suelen visitar los principales monumentos de la ciudad (pregunta 17), aunque no vayan a los museos (pregunta 18) ni a eventos deportivos (pregunta 24), seguramente por problemas de disponibilidad de tiempo libre. Sin embargo, creen que se mantienen los estereotipos folklóricos y que los turistas no distinguen la cultura catalana de la del resto de España (pregunta 21), por lo que suelen comprar productos típicos de otras regiones españolas (pregunta 20). También opinan que las actividades que realicen estos turistas en la ciudad no es necesario que estén demasiado organizadas previamente (pregunta 7), ya que no suelen traer problemas a la comunidad local (pregunta 9).

Según su grado de relación profesional con esta actividad turística, los encuestados presentan significativas diferencias únicamente en el volumen de tránsito de estos buques por el puerto de Barcelona. Así, solamente los encuestados que no tenían ningún tipo de relación con el turismo opinaban que deberían reducirse los cruceros que atracan en el puerto (pregunta 8). Otras diferencias de opinión entre ambos colectivos, aunque mucho menores que la anterior, se encuentran en las preguntas sobre si Barcelona está reconocida como puerto líder de cruceros en el Mediterráneo (pregunta 1), como destino 
turístico urbano internacional (pregunta 2), el grado de beneficio económico de este turismo (pregunta 11), el nivel de consumo y compra de este turismo (preguntas 12 y 16), la orientación de los comercios más céntricos hacia los turistas (pregunta 15), el conocimiento de la cultura catalana (preguntas 21 y 25); todas ellas valoradas más positivamente por los encuestados que tienen alguna relación con el turismo. Por su parte, los encuestados sin ninguna relación profesional con la industria turística creen más que este turismo no trae problemas a la comunidad local (pregunta 9).

Sin embargo, pese a su evidente impacto económico positivo, este turismo también presenta algunas sombras. Así, sin olvidar que el $80 \%$ de la oferta global de cruceros está concentrada solamente en tres empresas transnacionales: Carnival Corporation (50\%), Royal Caribbean (25\%) y Genting Hong Kong (7\%) (Legoupil, 2013), lo que provoca una competencia desigual para las pequeñas y medianas navieras y consolida un mercado cautivo, sus actividades también tienen importantes consecuencias medioambientales, como la contaminación de las aguas marinas, la generación y deslocalización de residuos peligrosos, la contaminación atmosférica, la destrucción de la biodiversidad marina y la contribución al calentamiento climático a escala global (Fernández Miranda, 2012). También se producen otros impactos ambientales debido a la ampliación de las infraestructuras portuarias (Tang y Jang, 2013) y a la inexistencia de una legislación global al respecto (Lagoupil, 2013).

Además de los aspectos medioambientales, se trata de un tipo de turismo que suele utilizar navíos con banderas de conveniencia y con la domiciliación del barco en paraísos fiscales, por lo que muchas de estas embarcaciones no están sujetas al pago de impuestos y otras contribuciones, lo que evita el reparto entre los países de desembarco de los beneficios generados (Robertson, 2009). También, en el plano laboral, la creación de empleo no puede esconder las precarias condiciones existentes en este sector, con salarios relativamente bajos, periodos de trabajo continuados de hasta seis meses, jornadas de siete días por semana y de 10 a 14 horas diarias, rotación elevada del personal y escasa formación, según la Federación Internacional de Trabajadores del Transporte (ITF, 2014).

Así pues, la percepción de la población local, esté o no relacionada de algún modo con el turismo, muestra que los residentes dan mayor importancia actualmente a los beneficios económicos que a los costes que este turismo puede reportar a la comunidad, por lo que presentan una actitud hacia él muy positiva (hipótesis 2), aunque ya empiezan a aparecer voces discordantes respecto a su sostenibilidad futura (hipótesis 3), como las asociaciones ecologistas $\mathrm{y}$ algunas asociaciones de vecinos.

\section{Conclusiones}

En este trabajo se ha explicado la importancia actual del turismo de cruceros para Barcelona y se ha descrito su evolución desde una posición marginal en la propia economía de la ciudad antes de la celebración de los Juegos Olímpicos de 1992 hasta colocarse en una situación de liderazgo internacional en este 
sector. La investigación ha mostrado también la importancia de la cooperación entre los agentes públicos y privados en la promoción y desarrollo de este tipo de turismo en Barcelona, lo que ha mejorado las infraestructuras portuarias y urbanas, ha ampliado los servicios logísticos, ha facilitado el desembarco de las grandes compañías navieras y ha desarrollado una oferta hotelera variada.

También se ha confirmado la actitud favorable de los residentes barceloneses hacia este turismo, que valoran más los impactos positivos que genera este turismo que los negativos. Sin embargo, últimamente ya se empiezan a vislumbrar los riesgos que genera esta actividad, debidos especialmente a sus impactos socioculturales y medioambientales y a la excesiva "turistificación» de algunas zonas comerciales de la ciudad.

Teniendo en cuenta todas estas consideraciones, y para mantener esta posición privilegiada, los responsables de la promoción turística, las autoridades portuarias y las compañías que operan en la ciudad deberán estar atentos no solamente a las oportunidades que genera este turismo sino también a los riesgos que produce; así como tener en cuenta las percepciones y actitudes de los residentes, todo ello con el objetivo de garantizar el éxito y la sostenibilidad de dicho turismo y su competitividad internacional.

La investigación sobre este tipo emergente de turismo es necesaria porque es una actividad que, en principio, parece presentar unos impactos más positivos que negativos sobre la economía local, tal como se aprecia en el caso de Barcelona, lo que es un factor importante en la estrategia del desarrollo turístico sostenible de muchas ciudades de nuestro litoral.

Por otra parte, la principal limitación de este estudio es que se ha analizado una encuesta con una muestra de tamaño reducido y realizada durante un único mes, diciembre, incluyendo las fiestas de Navidad y Fin de Año, por lo que los resultados aquí obtenidos deberían completarse con otras encuestas realizadas durante el resto del año, y así poder sentar las bases para futuras investigaciones sobre este tema. De esta manera, dichos resultados podrían ser aplicados a otras destinaciones con problemáticas similares. Finalmente, también sería interesante conocer la opinión de los propios cruceristas, así como las de los responsables de las empresas que organizan estas actividades.

\section{Referencias bibliográficas}

AB (1992-2012). Anuari estadistic de la ciutat de Barcelona. Barcelona: Ajuntament de Barcelona.

Alemany, J. (2002). El port de Barcelona. Un passat, un futur. Barcelona: Port de Barcelona.

Andriotis, K. y Agiomirgianakis, G. (2010). «Cruise visitors' experience in a Mediterranean port of call». International Journal of Tourism Research, 12 (4), 390-404. $<$ http://dx.doi.org/10.1002/jtr.770>

APB (2006-2009). Memoria corporativa. Barcelona: Autoritat Portuària de Barcelona. Brida, J. G.; Pulina, M.; Riaño, E. y Zapata, S. (2013). "Cruise Passengers in a Homeport: A Market Analysis». Tourism Geographies, 15 (1), 68-87. $<$ http://dx.doi.org/10.1080/14616688.2012.675510> 
Brida, J. G.; Riaño, E. y Zapata, S. (2012). «Percepciones de los residentes acerca de los impactos del turismo de cruceros en la comunidad: un análisis factorial y de clústeres». Cuadernos de Turismo, 29, 79-107.

Brida, J. G. y Zapata, S. (2008). "The impacts of the cruise industry on tourism destinations». Sustainable tourism as a factor of local development. Monza, 7-9/11/2008. <http://ssrn.com/abstract=1298403> [consulta: 14 de septiembre de 2014].

Buzard, J. (1993). The beaten track: European tourism, literature, and the ways to culture, 1800-1918. Oxford: Oxford University Press.

<http://dx.doi.org/10.1093/acprof:oso/9780198122760.001.0001>

Castillo, A. e Ybarra, I. (2004). La naviera Ybarra. Sevilla: Ybarra y Cía.

Castillo, A. M.; Osuma, M. y López, T. (2012). «Percepción y actitudes del residente acerca del impacto del turismo en la Isla de Santiago (Cabo Verde)». Turydes, $5,{ }^{\circ}{ }^{\circ} 12$.

CLIA (2014). CLIA's 2013 Cruise Market Profile: Report of Findings. Fort Lauderdale: Cruise Lines International Association.

Comín, E. (1990). «Validación de encuestas». Aten Primaria, 7, 386-390.

Cuellar-Río, M. y Kido-Cruz, M. T. (2008). «Perfil y análisis del gasto de crucerista. El caso de Bahías de Huatulco (México)». Cuadernos de Turismo, 22, 47-78.

Duman, T. y Mattila, A. S. (2005). "The Role of Affective Factors on Perceived Cruise Vacation Value». Tourism Management, 26 (3), 311-323. <http://dx.doi.org/10.1016/j.tourman.2003.11.014>

Dwyer, L. y Forsyth, P. (1998). «Economic significance of cruise». American of Tourism Research, 23, 393-413. <http://dx.doi.org/10.1016/S0160-7383(97)00098-4>

ECC (2012). Informe anual de contribuciones a la economía europea 2012. Bruselas: European Cruise Council.

Esteve, R. (1998). «El turismo de cruceros». En: La actividad turística española. Madrid: Asociación Española de Expertos Científicos, p. 627-636.

Euroxpress (2014). El turismo de mar y de costa, una de las joyas de la corona de la creación de empleo. <https://www.facebook.com/permalink.php?id=116321155094458\&story_ fbid $=799323226748762>$ [consulta: 4 de noviembre de 2014].

Fernández Fúster, L. (2009). "Acontecer del turismo de cruceros en Cuba y sus potencialidades para el mercado norteamericano». Turydes, 2, $\operatorname{art}^{\circ} 6$.

Fernández Miranda, R. (2012). Lo que hunden mientras flotan. Auge y análisis crítico del turismo de cruceros en la globalización. Barcelona: Alba Sud.

Garay, L. A. y CÀnoves, G. (2009). «El desarrollo turístico en Cataluña en los dos últimos siglos: una perspectiva transversal». Documents d'Anàlisi Geogràfica, 53, 29-46.

- (2012). "Turismo de cruceros en Barcelona. De la marginalidad al liderazgo internacional». Boletín de la Asociación Española de Geógrafos, 60, 253-271.

Harril, R. (2004). «Residents' attitudes toward tourism development: a literature review with implications for tourism planning». Journal of Planning Literature, 18 (1), 251-266.

<http://dx.doi.org/10.1177/0885412203260306>

Henthorne, T. L. (2000). «An analysis of expenditures by cruise ship passengers in Jamaica». Journal of Travel Research, 38 (3), 246-250. <http://dx.doi.org/10.1177/004728750003800306>

Hosteltur (2014). Informe anual 2013. Madrid: Hosteltur.

ITF (2014). Annual Raport 2013. Londres: International Transport Workers' Federation Press. 
Kester, J. G. C. (2002). «Cruise tourism». Tourism Economics, 9 (3), 337-350. <http://dx.doi.org/10.5367/000000003101298439>

Lawton, L. J. y Butler, R. (1987). «Cruise ship industry-patterns in the Caribbean 1880-1986». Tourism Management, 8 (4), 329-343. <http://dx.doi.org/10.1016/0261-5177(87)90091-4>

Legoupil, T. (2013). «Los conflictos que genera el turismo de cruceros en Barcelona y otros puertos mediterráneos». Biblio 3W. Revista Bibliográfica de Geografía y Ciencias Sociales, núm. 1.049.

Montero, L. (1996). «Estrategias de los puertos base de cruceros en el Mediterráneo». Boletín de Puertos del Estado, 35, 16-20.

Monterrubio, J. C. (2008). «Residents' perception of tourism: a critical theoretical and methodological review». Ciencia Ergo Sum, 15 (1), 35-44.

Murias, R. (2002). La industria del crucero en el siglo XXI. Implicación en los puertos españoles y perspectivas de futuro. Barcelona: Universitat Politècnica de Catalunya.

Nueva Tribuna (2014). «Barcelona bate récords en cruceros mientras oculta los graves problemas de contaminación del aire». Nuevatribuna.es.

<http://www.nuevatribuna.es/articulo/medio-ambiente/barcelona-bate-records-cruceros-mientras-oculta-graves-problemas-contaminacion-aire /20141014182713108214.html> [consulta: 12 de diciembre de 2014].

OMT (2008). Turismo de cruceros: situación actual y tendencias. Madrid: Organización Mundial del Turismo.

Paniagua, A. (2005). «El mercado de los cruceros en España. Una perspectiva histórica (1994-2004)». Estudios Turísticos, 165, 131-160.

Prat , J. M. y CÀnoves , G. (2012). «El patrimonio industrial como dinamizador del territorio. El caso del ecomuseo La Farinera, en Castelló d'Empúries (Cataluña)». Documents d'Anàlisi Geogràfica, 58 (1), 79-100.

Robertson, G. (2009). Informe sobre turismo en crucero 2008. Hamburgo: Lighthouse Foundation - Fundación para los Mares y los Océanos.

Silvestre, A., Santos, C. y Ramalho, C. (2008). «Satisfaction and behavioral intentions of cruise passengers visiting the Azores». Tourism Economics, 14 (1), 169-184. <http://dx.doi.org/10.5367/000000008783554802>

TANG, L. y Jang, S. (2013). "The Evolution from Transportation to Tourism: The Case of the New York Canal System». Tourism Geographies, 12 (3), 435-459. <http://dx.doi.org/10.1080/14616688.2010.494683>

Tімотну, D. (2006). Cruises, supranationalism and border complexities. Oxfordshire: CABI. <http://dx.doi.org/10.1079/9781845930486.0407>

Torres, J. M. y NieTo, E. (2014). "Ciudades y cruceros. Encuentros turísticos emocionalmente arriesgados». Comunicación presentada en el Seminario Internacional de Innovación y Competitividad en Áreas Turísticas. Alicante, Universidad de Alicante, del 5 al 7 de noviembre.

Towner, J. (1985). «The Grand Tour: A key phase in the history of tourism». Annals of Tourism Research, 12 (3), 297-333.

<http://dx.doi.org/10.1016/0160-7383(85)90002-7>

Turisme de Barcelona (2014). Estadístiques de turisme a Barcelona 2013. Barcelona: Barcelona Turisme.

Wood, R. E. (2000). "Caribbean cruise tourism: Globalization at sea». Annals of Tourism Research, 27 (2), 345-370. <http://dx.doi.org/10.1016/S0160-7383(99)00073-0> 